\title{
A Study on Insulation Characteristics of Glass Wool and Mineral Wool Coated with a Polysiloxane Agent
}

\author{
Chan-Ki Jeon, ${ }^{1}$ Jae-Seong Lee, ${ }^{1}$ Hoon Chung, ${ }^{2}$ Ju-Ho Kim, ${ }^{1}$ and Jong-Pil Park ${ }^{1}$ \\ ${ }^{1}$ Department of Urban Engineering, Incheon National University, 119 Academy-ro, Yeonsu-gu, Incheon 406-772, Republic of Korea \\ ${ }^{2}$ SunHan M↔T, 663-13 Gojan-dong, Namdong-gu, Incheon 405-818, Republic of Korea \\ Correspondence should be addressed to Jong-Pil Park; jp@inu.ac.kr
}

Received 10 October 2016; Accepted 28 December 2016; Published 30 January 2017

Academic Editor: Patrice Berthod

Copyright (c) 2017 Chan-Ki Jeon et al. This is an open access article distributed under the Creative Commons Attribution License, which permits unrestricted use, distribution, and reproduction in any medium, provided the original work is properly cited.

\begin{abstract}
The insulation in buildings is very important. Insulation used in the building is largely divided into organic and inorganic insulation by its insulation material. Organic insulation materials which are made of Styrofoam or polyurethane are extremely vulnerable to fire. On the other hand, inorganic insulation such as mineral wool and glass wool is very weak with moisture, while it is nonflammable, so that its usage is very limited. Therefore, this study developed moisture resistance applicable to mineral wool and glass wool and measured the thermal conductivity of the samples which are exposed to moisture by exposing the product coated with moisture resistance and without moisture resistance to moisture and evaluated how the moisture affects thermal conductivity by applying this to inorganic insulation.
\end{abstract}

\section{Introduction}

The issues of saving energy and reducing carbon dioxide emissions are concerns and important research projects in all countries. For this, the product development which maximized energy efficiency has been in progress and, in recent years, the research on the new insulation material development such as VIP (Vacuum Insulation Panels) using fumed silica and GFP (Gas-Filled Panels) using Argon (Ar), krypton $(\mathrm{Kr})$, and xenon $(\mathrm{Xe})$ gases which have a lower thermal conductivity than air has been actively progressed $[1,2]$.

Insulation boards are being used in various areas such as modern architecture and other industries, and these insulation boards are manufactured and used in various forms [3]. However, most of insulation is synthetic insulation in a foam type, where porosities are created inside of the product, fiber type insulation which uses glass wool or mineral wool in a nonwoven fabric type made from fabric material and board products which use inorganic binders such as cement with perlite and ceramic ball [4].

While insulation can be classified by raw material, type, and purpose of use, it is generally classified by material.
According to material, insulation can be divided into organic insulation and inorganic insulation. In the case of organic insulation, it has an excellent thermal performance, absorption, and workability, so that it occupies more than $90 \%$ of the domestic market; however, in case of fire, Styrofoam and urethane have less than 5 seconds in ignition time and the time taken for flame spread is 50 seconds, so that fire rapidly spreads and toxic gases generated during combustion such as formaldehyde, ethylene cyanide $(\mathrm{CH}=\mathrm{CHCN})$, hydrochloric acid gas, and cyanide gas are very critical to the human body [5].

In the case of inorganic insulation, it has an excellent fire resistance characteristic but its absorbability is very high, so that it has a drawback in that its insulation performance is poor [6]. While the thermal conductivity of air is $0.026 \mathrm{~W} / \mathrm{mk}$ [7], water has $0.598 \mathrm{~W} / \mathrm{mk}$ which is 23 times the thermal conductivity of air [8]. And also ice has $1.9 \mathrm{kcal} / \mathrm{mh}^{\circ} \mathrm{C}$ of thermal conductivity which is about 90 times or more the thermal conductivity of air, so that the water content of material can be the most influencing element determining thermal conductivity [9].

While thermal conductivity change in insulation material by water absorption has been widely reported, the research 


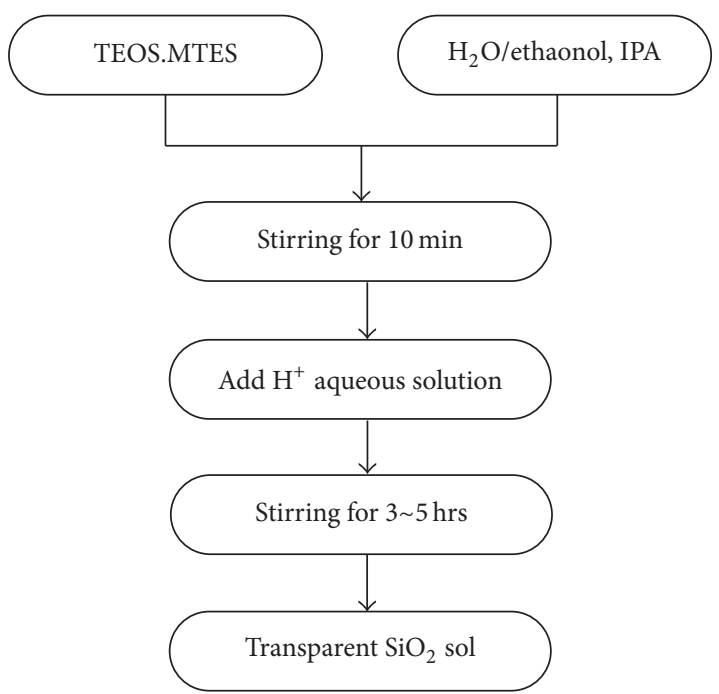

FIGURE 1: Synthesis of silica sol

for retaining insulation effect has not been reported, so that this study developed a moisture resistance and confirmed the waterproof ability of inorganic insulation by processing inorganic insulation materials, glass wool and mineral wool, with the moisture resistance, exposing them to moisture and measuring the amount of moisture increase and thermal conductivity [10-12].

In particular, this study measured the process where the heat on the surface is transferred and the temperature chance of the surface occurs according to the water absorption of mineral wool and glass wool by utilizing a thermal imaging camera and observed the effect and process that moisture does on insulation material [13].

\section{Experimental Device and Test Methods}

2.1. Experimental Device and Specimen. While there are comparative thermal conductivity measuring methods such as thermal conductivity heat flow meter and hot wire method [14], this study tested thermal conductivity measurement according to the KS L 9016 Test and the test was made by using a thermal conductivity meter (HFM-436) by thermal conductivity heat flow method. The glass wool and mineral wool used in this study used Korea KCC products. And the specimen size is $300 \times 300 \times 50 \mathrm{~mm}$ per KS L 9016, KS F 4714 test standard. Regarding the measurement of specimen, the thickness of the specimen was measured precisely and the thermal conductivity was measured in a place where surrounding temperature around the experiment space was kept constant. The thermal conductivity coefficient of the measured specimen was calculated by Fourier's law of heat conduction or the following equation [15]:

$$
\frac{d Q}{d T}=-A k \frac{\delta T}{\delta x}
$$

where $d Q / d T$ is heat flow rate/heat flux density $\left[\left(\mathrm{J} / \mathrm{m}^{2} \mathrm{~s}\right)\right]=$ [Watt $/ \mathrm{m}^{2}$ ], - indicates that heat flow direction is the direction

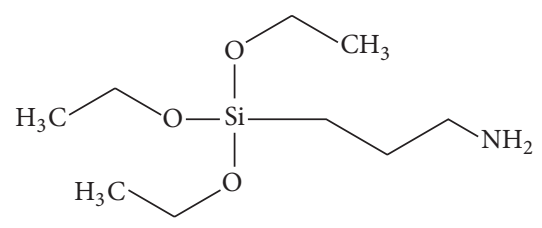

(a)<smiles>CCO[SiH2]CC[C+][C+][CH-][CH+](F)(F)F</smiles>

(b)

FIGURE 2: Silane compound constitutional formula: (a) 3-aminopropyltriethoxysilane; (b) tridecafluoro-1,1,2,2-tetrahydrooctyl-1-triethoxysilane.

cooling, $A$ is cross section $\left(\mathrm{m}^{2}\right), k$ is thermal conductivity $[\mathrm{J} /(\mathrm{msK})]$, and $\delta T / \delta x$ is temperature gradient (heat flow driving force) $(\mathrm{K} / \mathrm{m})$.

When looking at (1), the amount of heat conduction by unit time is proportional to the cross-sectional area contacting with the temperature difference and is inversely proportional to the distance.

2.2. Preparation of Moisture Resistance. The moistureresistant liquid in this study used nanosilicate which is manufactured in-house and fluoroalkylsiloxane compound and its preparation process is as follows [16].

2.3. Preparation of Silica Sol. Ethanol $1.4 \mathrm{~kg}(29.8 \mathrm{~mol})$ and concentrated hydrochloric acid $30 \mathrm{~g}(0.3 \mathrm{~mol})$ are put into water $3.0 \ell$ and mixed and then a mixed solution of tetraethoxysilane $2.08 \mathrm{~kg}(10 \mathrm{~mol})$ and methyltriethoxysilane $178 \mathrm{~g}(1.0 \mathrm{~mol})$ is added. Then, silica sol solution is obtained by stirring for 4 hours at room temperature. This process was confirmed by SEM and nanoparticle size analyzer and the reaction formula is as follows (Figure 1) [17].

\subsection{Preparation of Organosiloxane Containing Fluorinated} Alkyl Group. Tridecafluoro-1,1,2,2-tetrahydrooctyl-1-triethoxysilane $2.25 \mathrm{~kg}(5 \mathrm{~mol})$ is added to $3.0 \mathrm{~kg}$ of purified water and then aminopropyltriethoxysilane $1.10 \mathrm{~kg}(5 \mathrm{~mol})$ is added slowly. While stirring this solution, acetic acid $60 \mathrm{~g}(1 \mathrm{~mol})$ is added and stirred for 8 hours and then tridecafluoro-1,1,2,2tetrahydrooctyl-1-triethoxysilane (fluorine organosiloxane) is made (see Figure 2).

The reaction between tridecafluoro-1,1,2,2-tetrahydrooctyl-1-triethoxysilane and 3-aminopropyltriethoxysilane was confirmed with FT-IR.

2.5. Preparation of Fluoroalkylsiloxane Moisture Resistance (SH-AF). $10 \%$ silica sol of $100 \mathrm{~mL}$ solution and $10 \%$ organosiloxane of $100 \mathrm{~mL}$ are added and mixed into $800 \mathrm{~mL}$ of purified water and then $1,000 \mathrm{~mL}$ moisture-resistant solution is prepared.

2.6. Applying Moisture Resistance. When it comes to the samples for measuring the thermal conductivity, the glass wool and mineral wool specimens with $300 \times 300 \times 50 \mathrm{~mm}$ 
TABLE 1: Moisture absorption amount of mineral wool before and after SH-AF treatment.

\begin{tabular}{lcc}
\hline Classification & Weight of the sample before SH-AF coating & Weight of the sample after SH-AF coating \\
\hline Before humidification $(\mathrm{g})$ & 6.3 & 6.6 \\
After humidification $(\mathrm{g})$ & 6.58 & 6.7 \\
Water content $(\mathrm{g})$ & 0.28 & 0.1 \\
Percentage of moisture content (\%) & 4.18 & 1.49 \\
\hline
\end{tabular}

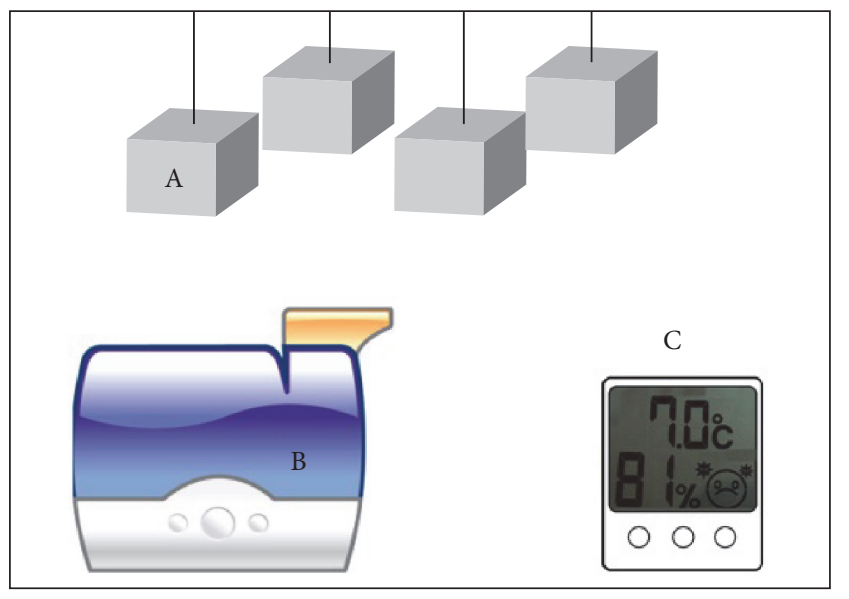

A: Glass wool

B: Humidifier

C: Hygrometer

FIgURE 3: Schematic drawing of the whole experimental set-up.

size are impregnated in the fluoroalkylsiloxane solution for 3 seconds and then are prepared by drying for 3 hours at $100^{\circ} \mathrm{C}$.

When it comes to the samples for measuring the absorption rate, they are created into $50 \times 50 \times 50 \mathrm{~mm}$ size to facilitate the humidification experiment and then they are impregnated in the fluoroalkylsiloxane solution for 3 seconds and then are prepared by drying for 3 hours at $100^{\circ} \mathrm{C}$.

The comparison was done by SEM to compare between the samples with the fluoroalkylsiloxane treatment and the samples with no fluoroalkylsiloxane treatment.

2.7. Absorption Measurement. While there are a pouring method and spray method to supply water for the measurement of the amount of absorption between mineral wool and glass wool samples with a coating and without a coating and due to the thermal conductivity change by absorption and the temperature change transmitted to the surface, this study supplied water by placing a humidifier in an acrylic box of $500 \mathrm{~mm}$ in length, width, and height as shown in Figure 3, leaving the sample for 4 hours with the hygrometer, indicating more than $90 \%$ in humidity.

2.8. Thermal Imaging Camera Measurement. In order to observe the diffusion of heat through thermal conductivity and a thermal imaging camera depending on the water supply method and water content of glass wool and mineral wool insulation materials, a hot plate was used as heat source and the temperature was fixed at $80^{\circ} \mathrm{C}$. Regarding the thermal imaging camera, the products of PI and FL companies were used for the observation. At the time, the camera was fixed in order to measure the temperature of the surface and the middle of the sample.

\section{Results}

\subsection{Preparation of Fluoroalkylsiloxane}

3.1.1. Preparation of Silica Sol. The observation result with TEM (Transmission Electron Microscopy) by diluting synthesized $\mathrm{SiO} 2$ sol with ethanol in a ratio of $14: 1$ showed that spherical $\mathrm{SiO} 2$ nanoparticles with approximate size of $15 \mathrm{~nm}$ were created (Figure 4) similar to the particle size analysis. The measurement result of synthesized silica sol by the particle size analyzer (Zetasizer Nano ZS90, Malvern) confirmed that the average particle size was $14.6 \mathrm{~nm}$ and very uniform sizes of $\mathrm{SiO} 2$ nanoparticles were synthesized within $\pm 0.549 \mathrm{~nm}$ in the particle size distribution.

3.2. SEM Photos. The test result shows that $\mathrm{SH}-\mathrm{AF}$ has been well coated to mineral wool and glass wool as shown in Figure 5 which compares the sample with moisture resistance and the sample with no moisture resistance with SEM photos.

3.3. Thermal Conductivity. The measurement result of thermal conductivity for each test piece shows that thermal conductivity of typical mineral wool is $0.035 \mathrm{~W} / \mathrm{mk}$ and thermal conductivity of mineral wool with $\mathrm{SH}-\mathrm{AF}$ processed is $0.0344 \mathrm{~W} / \mathrm{mk}$, so that it becomes lower. Also, in case of glass wool, thermal conductivity of typical glass wool is $0.0343 \mathrm{~W} / \mathrm{mk}$ and thermal conductivity of glass wool with $\mathrm{SH}$-AF processed is $0.0329 \mathrm{~W} / \mathrm{mk}$, which means it becomes a little bit lower in the same way as mineral wool. So, based on these results, it was confirmed that $\mathrm{SH}-\mathrm{AF}$ treatment makes thermal conductivity lower so that insulation performance increases a little bit [18] (see Figure 6).

3.4. Water Absorption Amount of Specimen and Thermal Conductivity of Mineral Wool with Moisture. Weight change shown from the moisture absorption measurement after supplying moisture for 4 hours through a humidifier is shown in Tables 1 and 2. Typical mineral wool absorbed $4.18 \%$ of moisture and mineral wool with coated $\mathrm{SH}-\mathrm{AF}$ did $1.49 \%$ of moisture. Typical glass wool absorbed $8.67 \%$ of moisture and one with coated SH-AF did only $0.46 \%$ of moisture. This result confirms that $\mathrm{SH}-\mathrm{AF}$ moisture resistance developed 


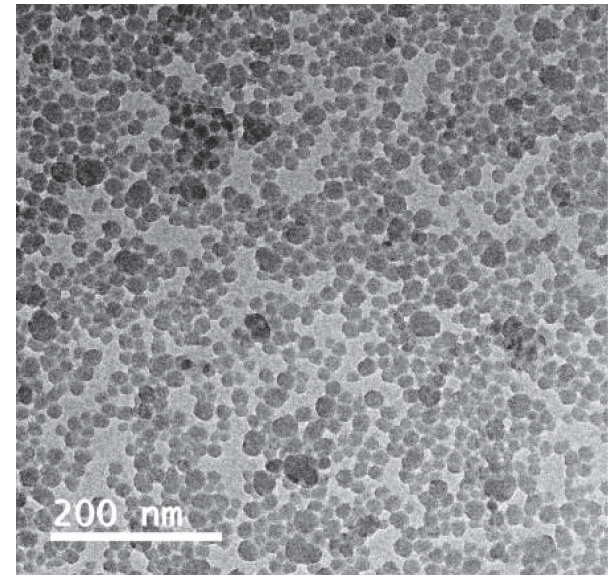

(a)

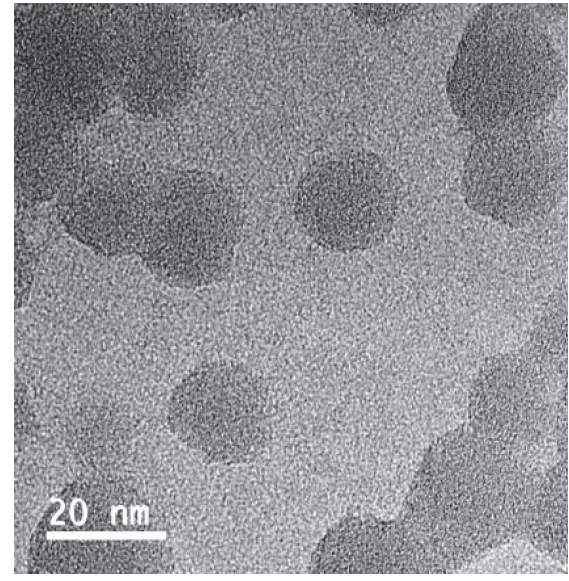

(b)

FIgURE 4: TEM of nanosilica: (a) $200 \mathrm{~nm}$ silica TEM picture; (b) $20 \mathrm{~nm}$ silica TEM picture.

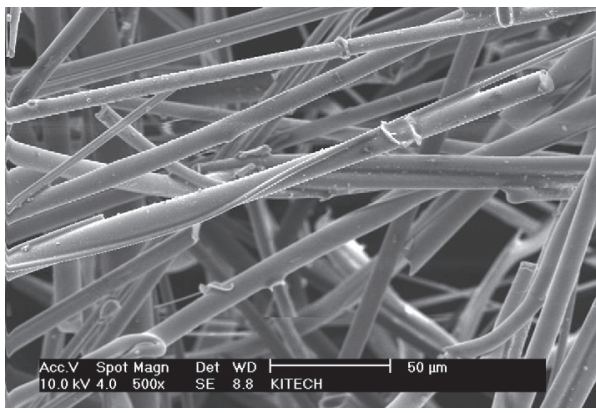

(a)

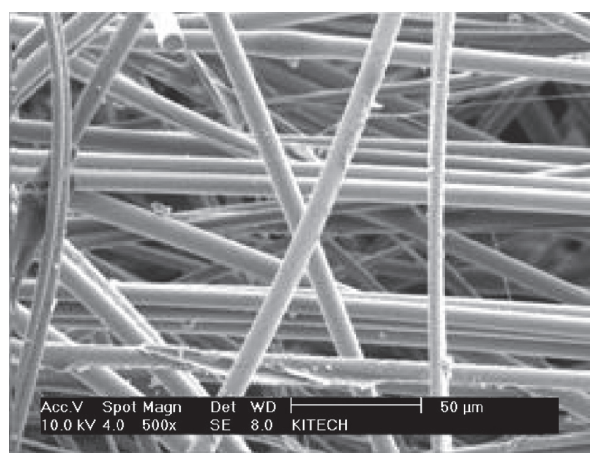

(c)

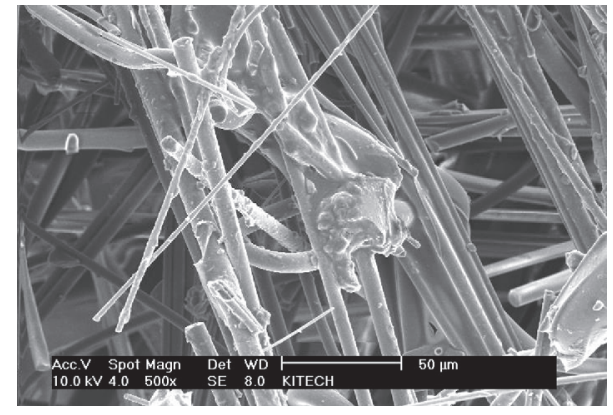

(b)

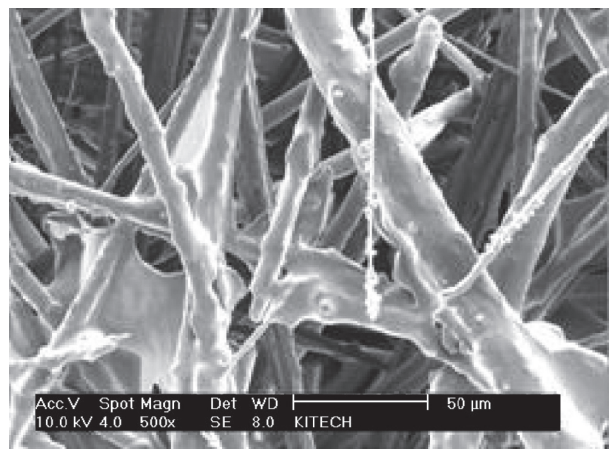

(d)

FIGURE 5: SEM of mineral wool and Glass wool: (a) mineral wool; (b) mineral wool coated with SH-AF; (c) glass wool; (d) glass wool coated with SH-AF.

TABLE 2: Moisture absorption amount of glass wool before and after SH-AF treatment.

\begin{tabular}{lcc}
\hline Classification & Weight of the sample before SH-AF coating & Weight of the sample after SH-AF coating \\
\hline Before humidification $(\mathrm{g})$ & 4.50 & 4.38 \\
After Humidification $(\mathrm{g})$ & 4.89 & 4.40 \\
Water content $(\mathrm{g})$ & 0.39 & 0.02 \\
Percentage of moisture content (\%) & 8.67 & 0.46 \\
\hline
\end{tabular}




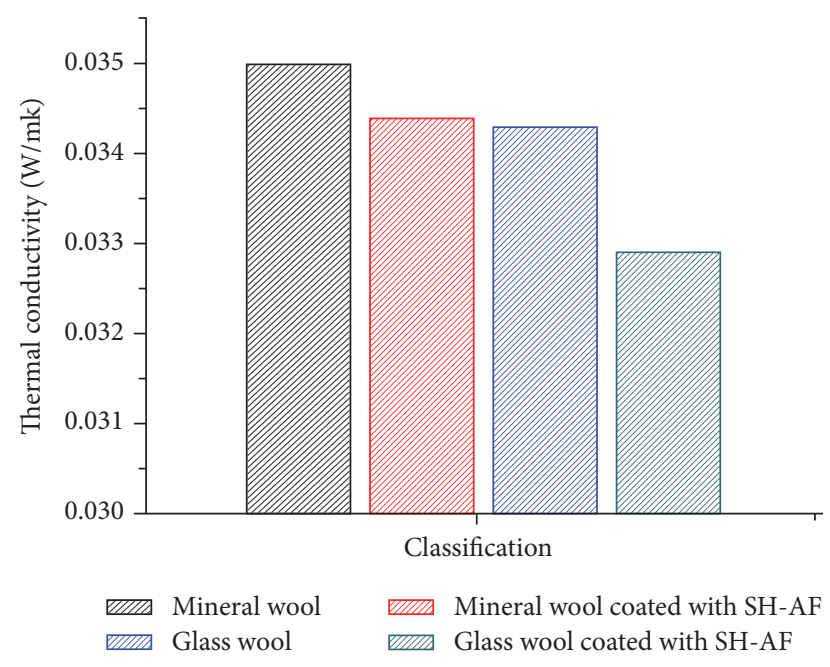

FIGURE 6: Thermal conductivity of mineral wool and glass wool.

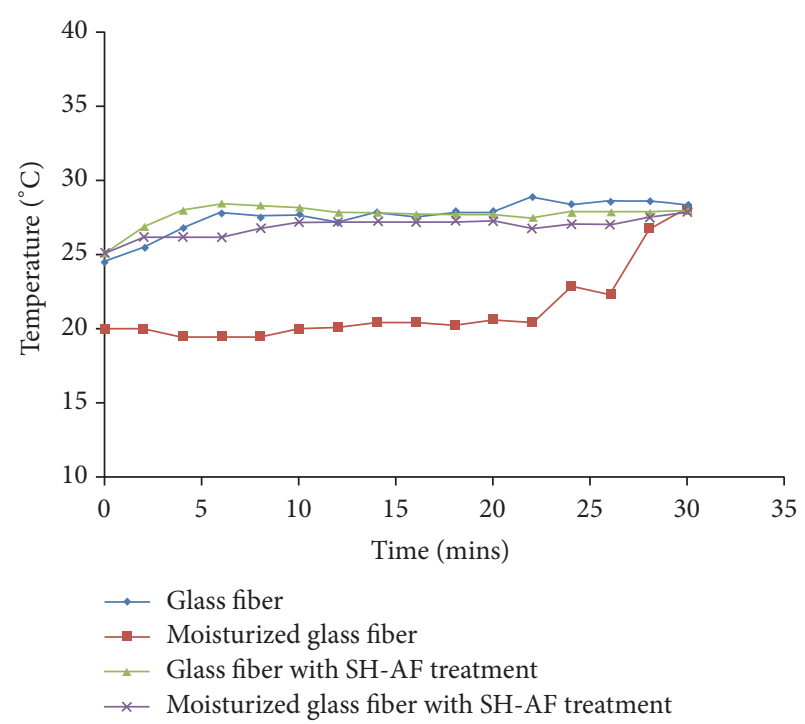

FIGURE 7: Surface temperature change in glass fiber before and after moisturizing.

in this study can be applied to existing inorganic insulation materials.

It was found that the glass wool with moisture has $0.136 \mathrm{~W} / \mathrm{mk}$ in thermal conductivity so that thermal conductivity increases 4 times compared to $0.0343 \mathrm{~W} / \mathrm{mk}$ shown in typical glass wool.

3.5. Temperature Change in Inorganic Material. Figure 7 shows the glass wool sample with moisture resistance ( $\mathrm{SH}-$ $\mathrm{AF})$ treatment and one without that and the temperature change of the glass wool sample with moisture resistance ( $\mathrm{SH}-\mathrm{AF})$ treatment and one without that. After moisture is supplied for 4 hours through a humidifier for each sample [19], the temperature change on the side and top surface of insulation material was checked with a thermal imaging camera. The result shows that while the glass wool with moisture resistance (SH-AF) treatment has no big change in surface temperature, the temperature arises suddenly after being kept low in the beginning with the glass wool specimen with no moisture-resistant coating. It can be understood that the moisture in inorganic insulation material evaporates and then the performance of insulation material is reduced. It can be found that the moisture-resistant (SH-AF) treatment prevents thermal conductivity of the sample to rapidly drop by moisture [20].

\section{Conclusion}

In this paper, the temperature change of insulation material was measured after applying fluoroalkylsiloxane moisture resistance developed in-house to typical inorganic insulation materials and the condition similar to the summer monsoon period was applied to inorganic insulation material by the humidification method as a way of moisturizing in the test. The experimental results are as follows:

(1) Inorganic insulation materials such as glass fiber or mineral wool are extremely vulnerable to moisture so that they absorbed water $4 \sim 8 \%$ of their weight and thermal conductivity increased more than 4 times so that it is difficult to expect a proper performance as insulation in a high humidity area.

(2) Fluoroalkylsiloxane (SH-AF) moisture resistance developed in this study suppressed moisture absorption when applied to inorganic insulation so that it can prevent thermal conductivity from being increased by moisture which is a drawback of inorganic insulation material.

(3) In previous researches, pouring method or spray method was used for testing as a method of supplying water to inorganic insulation material, but when evaluating the impact that moisture makes on insulation performance, it is effective to evaluate the effect of moisture with a more realistic moisturizing method so that setting up a standard test method is necessary.

(4) With the conventional testing device for measuring the thermal conductivity, thermal conductivity of insulation material with moisture cannot be measured so that hot wire method was used to measure thermal conductivity of insulation material with moisture. Therefore, a standard method for measuring change in thermal conductivity by moisture absorption in insulation material should be presented.

\section{Competing Interests}

The authors declare that they have no competing interests.

\section{Acknowledgments}

This study was performed by the research funding from Korea Institute of Energy Technology Evaluation and Planning (Project no. 20132020102400). 


\section{References}

[1] G. Ma, L. Huang, L. Yan, H. Wang, and P. Yin, "Flexural and Thermal Properties of Novel Energy Conservation Slotted Reinforced Concrete Beams," Advances in Materials Science and Engineering, vol. 2016, Article ID 4642810, 14 pages, 2016.

[2] K. S. Al-Jabri, A. W. Hago, A. S. Al-Nuaimi, and A. H. Al-Saidy, "Concrete blocks for thermal insulation in hot climate," Cement and Concrete Research, vol. 35, no. 8, pp. 1472-1479, 2005.

[3] G. Rosace, E. Guido, C. Colleoni, and G. Barigozzi, "Influence of textile structure and silica based finishing on thermal insulation properties of cotton fabrics," International Journal of Polymer Science, vol. 2016, Article ID 1726475, 10 pages, 2016.

[4] E. Barreira and V. P. De Freitas, "The effect of nearby obstacles in surface condensations on external thermal insulation composite systems: experimental and numerical study," Journal of Building Physics, vol. 37, no. 3, pp. 269-295, 2014.

[5] S. Farhan, R. Wang, H. Jiang, and K. Li, "Use of waste rigid polyurethane for making carbon foam with fireproofing and anti-ablation properties," Materials \& Design, vol. 101, pp. 332$339,2016$.

[6] K. Kadoya, N. Matsunaga, and A. Nagashima, "Viscosity and thermal conductivity of dry air in the gaseous phase," Journal of Physical and Chemical Reference Data, vol. 14, no. 4, pp. 947970, 1985.

[7] J. V. Sengers and J. T. R. Watson, "Improved international formulations for the viscosity and thermal conductivity of water substance," Journal of Physical and Chemical Reference Data, vol. 15, no. 4, pp. 1291-1314, 1986.

[8] A.-M. Kietzig, S. G. Hatzikiriakos, and P. Englezos, "Ice friction: the effect of thermal conductivity," Journal of Glaciology, vol. 56, no. 197, pp. 473-479, 2010.

[9] C. Langlais, M. Hyrien, and S. Klarsfled, "Moisture migration in fibrous insulating material under the influence of a thermal gradient and its effect on thermal resistance," ASTM STP, vol. 779, pp. 191-206, 1928.

[10] B. Choi, I. Yeo, J. Lee, W. K. Kang, and T.-H. Song, "Pillarsupported vacuum insulation panel with multi-layered filler material," International Journal of Heat and Mass Transfer, vol. 102, pp. 902-910, 2016.

[11] F. Bisegna, B. Mattoni, P. Gori et al., "Influence of insulating materials on green building rating system results," Energies, vol. 9, no. 9, article 712, 2016.

[12] D. A. Kontogeorgos, G. K. Semitelos, I. D. Mandilaras, and M. A. Founti, "Experimental investigation of the fire resistance of multi-layer drywall systems incorporating Vacuum Insulation Panels and Phase Change Materials," Fire Safety Journal, vol. 81, pp. 8-16, 2016.

[13] S. Chudzik, "Measurement of thermal parameters of a heat insulating material using infrared thermography," Infrared Physics and Technology, vol. 55, no. 1, pp. 73-83, 2012.

[14] U. Hammerschmidt, J. Hameury, R. Strnad, E. Turzó-Andras, and J. Wu, "Critical review of industrial techniques for thermalconductivity measurements of thermal insulation materials," International Journal of Thermophysics, vol. 36, no. 7, pp. 15301544, 2015.

[15] J. Li and J. D. Lee, "Reformulation of the Nosé-Hoover thermostat for heat conduction simulation at nanoscale," Acta Mechanica, vol. 225, no. 4-5, pp. 1223-1233, 2014.

[16] A. Chunglok, N. Muensit, and C. Daengngam, "Extreme Wetting-Resistant Multiscale Nano-/Microstructured Surfaces for Viscoelastic Liquid Repellence," Journal of Nanomaterials, vol. 2016, Article ID 9510156, 13 pages, 2016.

[17] F. Luo, Z. Shao, Y. Zhang, and X. Cheng, "Synthesis of paramagnetic iron incorporated silica aerogels by ambient pressure drying," Materials Chemistry and Physics, vol. 142, no. 1, pp. 113118, 2013.

[18] T. S. Yun, Y. J. Jeong, and K.-S. Youm, "Effect of surrogate aggregates on the thermal conductivity of concrete at ambient and elevated temperatures," The Scientific World Journal, vol. 2014, Article ID 939632, 9 pages, 2014.

[19] F. Stazi, F. Tittarelli, G. Politi, C. Di Perna, and P. Munafò, "Assessment of the actual hygrothermal performance of glass mineral wool insulation applied 25 years ago in masonry cavity walls," Energy and Buildings, vol. 68, pp. 292-304, 2014.

[20] W. Jiang, A.-M. Sha, J.-Z. Pei, and Z.-J. Wang, “Thermophysical properties and thermal resistance function of permeable asphalt concrete," Gongneng Cailiao/Journal of Functional Materials, vol. 43, no. 3, pp. 379-382, 2012. 

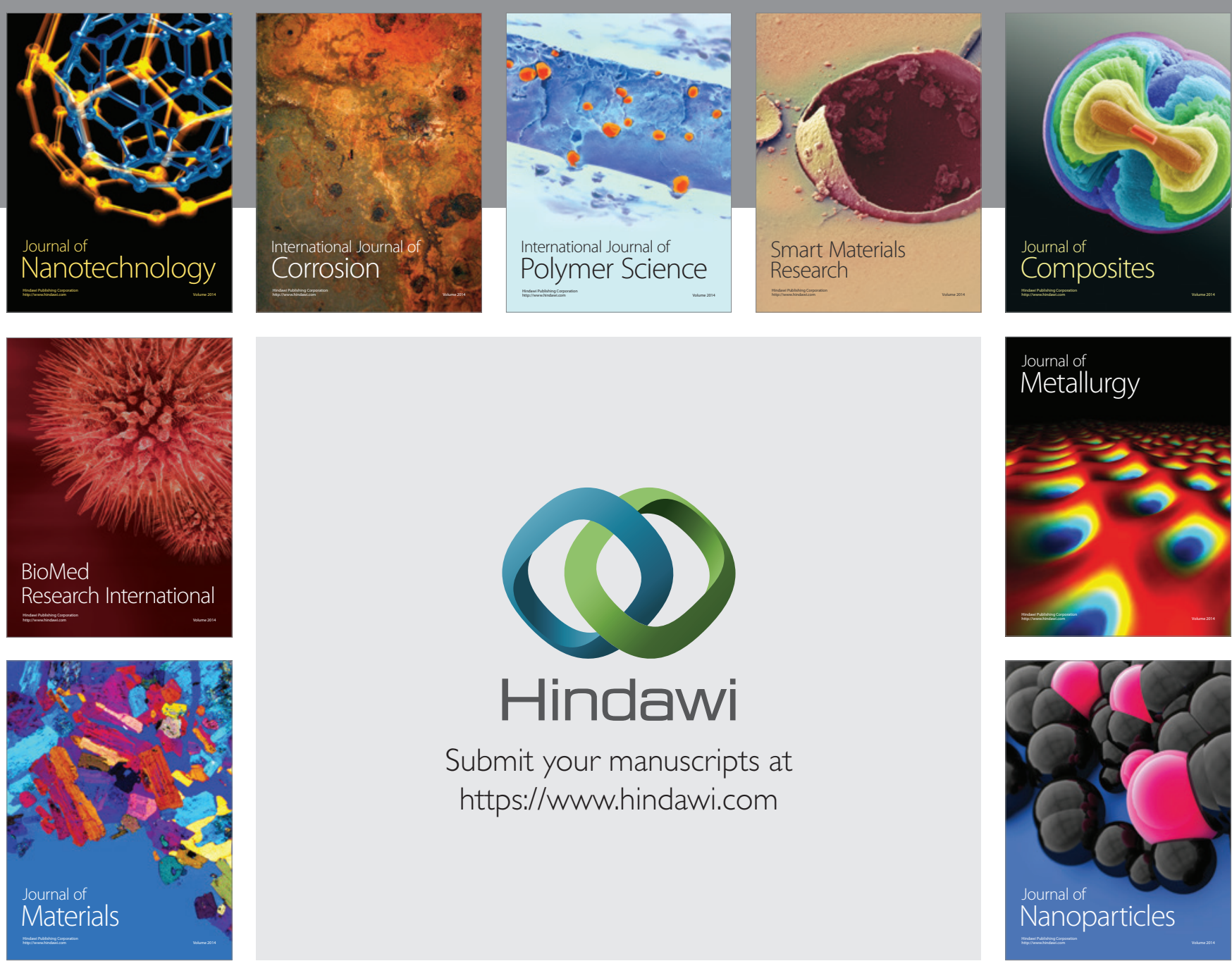

\section{Hindawi}

Submit your manuscripts at

https://www.hindawi.com

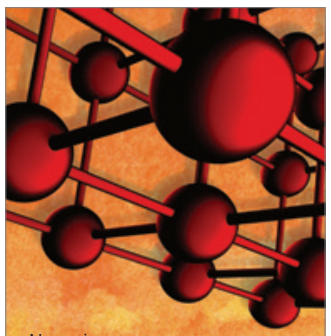

Materials Science and Engineering
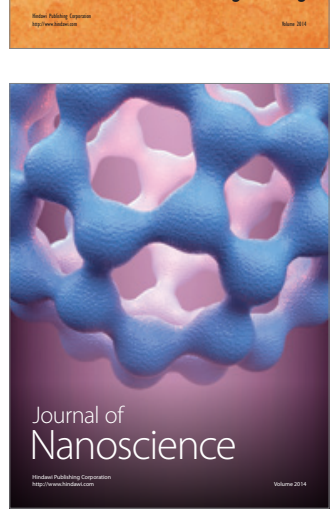
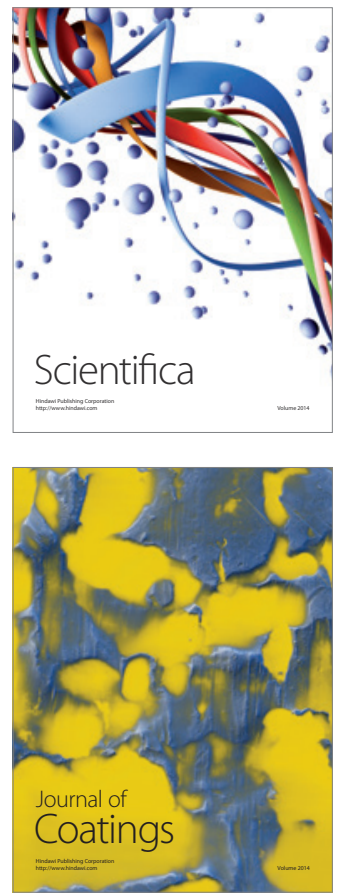
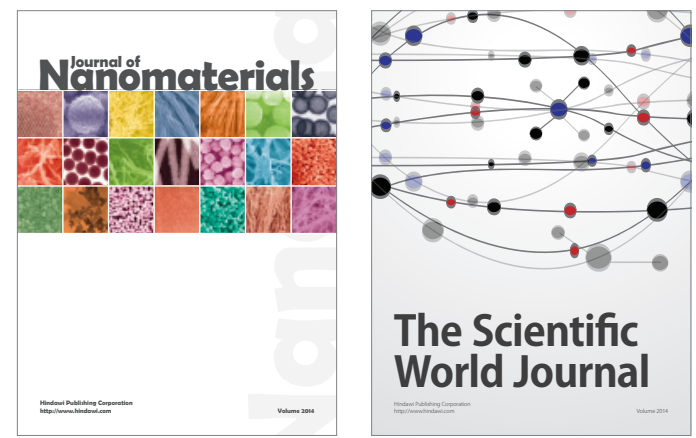

The Scientific World Journal
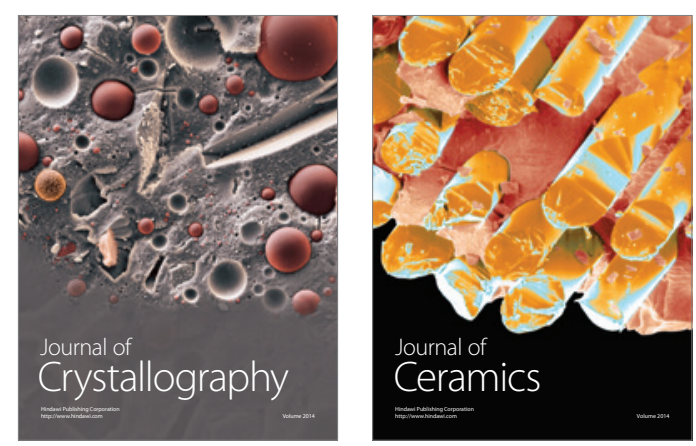
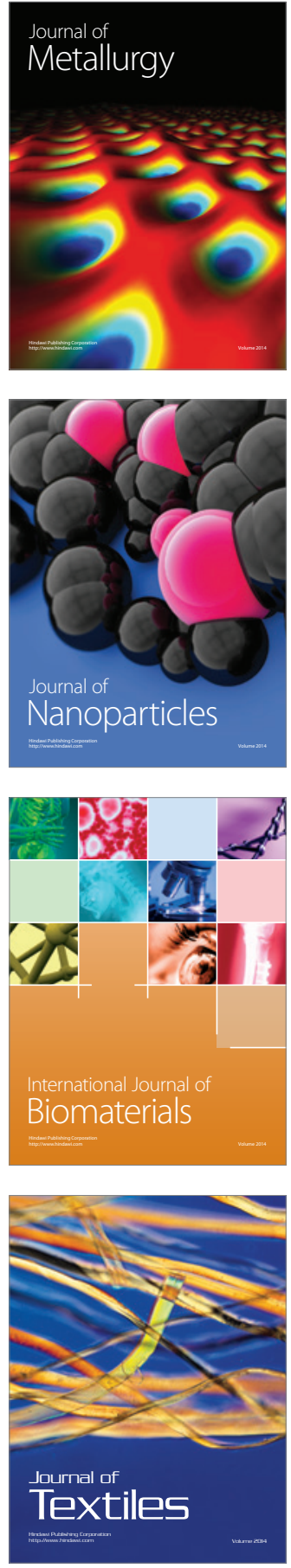